\title{
Five-year Surveillance of Malaria in Hotspot and Ivermectin Mass-drug Administration Zone of Amhara Regional State, Ethiopia
}

Chalachew Yenew ( $\square$ yenewdenku716@gmail.com)

Debre Tabor University https://orcid.org/0000-0003-1000-9220

\section{Sileshi Mulatu}

Bahir Dar University

Asaye Alamneh

Debre Tabor University

\section{Research}

Keywords: Malaria Trend, Ivermectin, Mass-drug Administration, Ethiopia

Posted Date: May 24th, 2021

DOI: https://doi.org/10.21203/rs.3.rs-546789/v1

License: (c) (i) This work is licensed under a Creative Commons Attribution 4.0 International License.

Read Full License 


\section{Abstract}

Objectives: Evaluate the five-year surveillance of malaria in the hotspot and Ivermectin mass-drug administration Zone of Amhara Regional State, Ethiopia.

Methods: - A descriptive prevalence study design was employed and incorporated 25 study health institutions into the survey using the purposive sampling technique. Data were obtained by the standard format of systematic evaluation of four surveillance units from January to August 2020 through observation, document review, and interviewing surveillance officers and focal persons using a semistructured Survey and generated the statistical analysis, tabular, and graphical output using the opensource statistical program R.

Results: - Average report fullness and aptness were $97.9 \%$ and $96 \%$ subsequently. The average annual malaria incidence rate declined in terms of place and time, from the year 2015 to 2019 with an average reduction rate of $5.5 \%$ and the average annual parasitic incidence rate was $52 \%$. The study identifies high endemicity of malaria due to no program-specific supportive supervision of public health emergency management and no routine data analysis.

Conclusions: This result revealed that the malaria incidence rate showed a remarkable decline. However, the annual parasitic incidence rate remains constant. The study also indicated that ivermectin did not affect malaria elimination. Hence, the districts and sub-city health offices should conduct regular surveillance data analysis, perform supportive supervision, avail budgets, and further laboratory investigations to investigate the effect of ivermectin on the parasites under laboratory conditions.

\section{Background}

Intestinal parasitic diseases like malaria are curable. But, life-threatening infections cause intense febrile sickness, which taints the human liver and red blood cells. It is transmitted from individual to individual by the chomp of the Anopheles mosquito, which chomps as it were at night. It is transferred to individuals via the chomps of Anopheles mosquitoes, called malaria vectors, taints with Plasmodium parasites (PPs) (World malaria report, 2019).

In 2018, assessed 228 million diseases of malaria occurred globally (95\% confidence interval (CI): 206258 million), in contrast with 251 million infectious in 2010 (95\% confidence interval (Cl): 231278 million) and 231 million infections in 2017 (95\% confidence interval (Cl): 211-259 million). In the 2018 World Health Organization (WHO) malaria report showed that the African Region (213 million or 93\%), followed by the South-East Asia Region with 3.4\% of the diseases and the Eastern Mediterranean Region with $2.1 \%$ and In 2018, there were an estimated 405000 mortality from malaria globally, compared with 416000 determining mortality in 2017, and 585000 in 2010 (The Carter Center, 2012).

Public health surveillance (PHS) is the continuing organized gathering, investigation, elucidation, and well-timed distribution of health-related information for activities and program evaluation. Sustain the 
decreased trouble of immediately reportable diseases (IRDs), eradicate the disease, and prevent its restitution are the goals (World Health Organization, 2018).

According to Ethiopia, malaria elimination strategy, mortality, and morbidity attributed to malaria declined significantly during the review period. Accordingly, death due to malaria declined by $67 \%$ from $0.9 / 100,000$ population to $0.3 / 100,000$ population at risk between 2016 and 2019 . Similarly, the annual parasite incidence (API) has declined by $37 \%$ from the $19 / 1000$ population to the $12 / 1000$ population between 2016 and 2019. The number of confirmed malaria cases has reduced by $47 \%$ between 2016 and 201 (Chala, 2020).

PHS of infectious diseases are recognized as the keystone of Public Health (PH) decision-making and practice. The data of PHS for monitoring the health status of the population, detecting diseases, and triggering actions are crucial (World Health Organization, 2018).

WHO recommended mass drug administration (MDA) of ivermectin as a potential tool to reduce malaria transmission to reach malaria vectors that feed on the temporal and spatial gaps left by core vector control interventions (Chaccour et al., 2017).

Information distributed by a PHS system used for immediate $\mathrm{PH}$ action, program planning and evaluation, and formulating research suggestions. PHS system developed to address a range of $\mathrm{PH}$ needs. They include a variety of data sources essential to PH action (German et al., 2001).

Malaria PHS predicts the maintenance of continuing watch over the status of malaria in a community. The primary purpose of PHS is to detect changes in trends or distribution of malaria and other vectorborne diseases to initiate investigation or control measures. Aims of malaria PHS is the prevention and control of malaria in the community (World Health Organization, 2013).

Conducting PHS is a clue for monitoring the efficacy and effectiveness of interventional programs in the health care system. Effective PHS systems are one of the basic strategies of national disease prevention and control programs. A PHS scheme serves two relevant roles; (i) untimely caution of likely threats to $\mathrm{PH}$ and (ii) program monitoring of the PHE, which could be emerging-definite or multiple-emergency in life (Anam et al., 2019). Therefore, the purpose of this study was to evaluate Five-year surveillance of Malaria in the hotspot and Ivermectin mass-drug administration Zone of Amhara Regional State, Ethiopia, 2020.

\section{Methods}

\section{Study design and period}

The institution-based prevalence study design was employed based on an "overview of evaluating PHS systems CDCs updated in the 2020 guideline for evaluating PHS system" as a framework for the evaluation to achieve the stated objective of the study from June 2020 to August 2020.

\section{Study unit}


The study units were woreda HOs and health facilities (HF). 25 study sources were included in the survey, including woreda $\mathrm{HOs}=5, \mathrm{HCs}=10$, and $\mathrm{HPs}=10$.

\section{Sample size and technique}

Awi zone is one of the zones in the Amhara Region that reported an uppermost figure of cases in the region during the study. We purposely selected to conduct the PHS system evaluation in this zone based on previous malaria hotspot and Ivermectin mass drug administration. A 25 health institutions (05 HO: 10 $\mathrm{HC}$ and each selected HC: $10 \mathrm{HP}$ ) included in the study using a purposive sampling method based on their malaria weekly report caseload and surveillance performances.

\section{Data collection and sources}

Data collection was done by face-to-face interviews using questionnaires/checklists. HO head and PHEM officers responded and observed the activities of surveillance and secondary data review. And the increasing or decreasing rate before and after ivermectin mass drug administration was measured by the annual parasitic incidence rate. And the population data used to determine the Annual parasitic incidence rate and the disease rate.

\section{Data management and analysis}

We coded and entered the data using Epi info 7. The analysis and statistical tabular and graphical output generated using the open-source statistical program R. The results were presented with text descriptions, graphs, and tables.

\section{Data Quality assurance}

We reviewed the data by $\mathrm{PH}$ experts who have worked in the PHS system. Some data was de-duplicated, especially the data from malaria monitoring charts, weekly PHEM reports, and year reports. We cleaned and analyzed data using Microsoft Office Excel 2016 and Epi-data to show report completeness, timeliness, and trends over time and the geographical distributions.

\section{Results}

According to the open-source statistical program R output, the average completeness of the weekly report collected from $2015-2019$ was $97.9 \%$ (95\% Cl: 96.2\%, 98.9\%). The average report timeliness was $96 \%$ (95\% Cl: 95.5\%, 97.7\%) (Fig. 1).

Figure 1: The open-source statistical program $\mathrm{R}$ graphical outputs of the timelines \& completeness of the reports of different woreda of Awi Zone, Amhara Regional State, Ethiopia, 2020.

The overall report completeness and timeliness rate was above the World Health Organization minimum goals (80\%) in the last five years (Fig. 1). The difference might be due to increasing awareness of the 
community and acceptance of the community, health extension workers, and other health providers of the PHS.

In this analysis, we were done a malaria incidence rate of the zone and woreda according to the previous 5-year data to identify the hotspot zone and woreda. The surveillance data from the year 2015-2019 provided that North Gondar (59.7), Awi (54.3), South Gondar (27.5), and West Gojam (26.3) per 1000 populations had the highest Annual average incidence rate (Fig. 2).

Figure 2: The open-source statistical program R Graphical output of malaria average annual parasitic incidence rate per 1000 population by zones in Amhara Region, Ethiopia, 2015-2019.

In the current study, the Awi zone is our study area because the second hotspot area and all woreda were Ivermectin mass drug administration targeted woreda from the Year 2015-2019 and malaria incidence rate (Table 1 and Fig. 3).

Table 1

The open-source statistical program $\mathrm{R}$ tabular outputs of the malaria incidence rate of the study area from 2015-2019, Awi zone, Amhara Region, Ethiopia.

\begin{tabular}{|lllllll|}
\hline s.no & Name of Woreda & $\mathbf{2 0 1 5}$ & $\mathbf{2 0 1 6}$ & $\mathbf{2 0 1 7}$ & $\mathbf{2 0 1 8}$ & $\mathbf{2 0 1 9}$ \\
\hline 1 & Ankesha & 35.70 & 22.70 & 11.33 & 7.30 & 7.26 \\
\hline 2 & Banja & 56.85 & 24.80 & 15.75 & 10.21 & 12.46 \\
\hline 3 & Chagni & 160.20 & 88.56 & 53.22 & 59.57 & 80.91 \\
\hline 4 & Dangila Ketama & 21.97 & 10.52 & 9.37 & 7.66 & 9.71 \\
\hline 5 & Dangila zuria & 28.06 & 16.42 & 12.41 & 8.18 & 13.85 \\
\hline 7 & Fagita lokoma & 30.01 & 14.88 & 9.67 & 8.81 & 4.82 \\
\hline 8 & Guagusa shekudade & 47.74 & 18.30 & 13.37 & 7.96 & 6.23 \\
\hline 10 & Injebra & 44.51 & 31.48 & 13.74 & 8.57 & 22.82 \\
11 & Jawi & 45.76 & 15.06 & 12.88 & 8.18 & 8.98 \\
\hline
\end{tabular}

No effect of Ivermectin on malaria elimination because the current API rate was still high. API reported from 2015-2019 was 0.52 (Fig. 4)

\section{Population under PHS}

PHEM targets all the population to be under surveillance for all the 21 diseases nationally and 22 diseases (include Lehimaniasis) in the Amhara region. All residents who selected Woreda of Awi Zone 
and Visited Woredas by 2020 covered in the study.

The national PHEM targets all the population in the country to be under PHS for all the 22 priority diseases. The Awi zone cascade the same structure, with a total population of 1,253,909 in 2020 with a population conversion factor from this $\mathbf{4 7 4 0 1 2}$ population under PHS.

\section{Current malaria test results}

PHEM reports of $2019 / 2020$ showed that 143,131 total malaria fever cases were examined by RDT or Microscopy regionally. Of which 43,131 confirmed malaria cases reported in the Awi zone. Of this 33,666 (78\%) and 9,465 (22\%) were Plasmodium falciparum and Plasmodium Vivax respectively. Malaria accounts for $16.6 \%$ of the total outpatient cases and $0.07 \%$ of the inpatient cases. Of the total malaria cases, 6,255 (14.5\%) were under-five children, $21 \%$ were people aged from $5-14.64 .5 \%$ were above 15 years old. However, there were $1(0.02 \%)$ deaths due to malaria. The death of pregnant mothers was 596 (1.5\%). During the same year, Awi Zone also reported 259,009 (35.6\% of the region) total malaria cases examined by RDT or Microscopy, of which 18,726 (58\%) from the zone confirmed (Fig. 5).

Figure 5: The open-source statistical program R graphical outputs of trends of confirmed malaria cases by woreda and WHO Epidemiologic week of Awi Zone, woreda, Amhara region Ethiopia, 2020.

A Zone reported 43,131 confirmed malaria cases reported from July 2019 to August 2020. A total of 41,959 and 8,793 confirmed malaria cases were reported from the woredas Ankesha, Banja, Changi, Guasgusa, and Zigem respectively to Awi Zone from July 2019 to January 2020 . Of these, 6,430 (73\%) cases were positive for Plasmodium falciparum. The 2020 PHEM report also showed 8793 confirmed malaria cases reported from 27 weeks up to WHO week 52.6430 (73\%) and $2363(27 \%)$ were plasmodium falciparum and plasmodium Vivax substantially.

\section{Discussion}

This study intended to evaluate the five-year surveillance of malaria in Awi zone, Amhara Region, Ethiopia, 2020 with the effect of ivermectin on malaria reduction by describing and measuring indicators.

We identified total Malaria cases of 259,009 outpatients, 43,131 in-patients, and one death recorded from 2011-2020 in Awi Zone. The most affected woredas in 2019 are Guasgusa Shekudad, Changi, and Zigem 314, 281, and 245 per 1000 subsequentially. Of all woredas in the Zone, Guasgusa Shekudad, Changi, and Zigem woredas were the most affected woredas from 2015-2020 (2,096 per 10000 population).

The analysis result showed that malaria occurred throughout the year. But, it declined from 340/1000 in 2015 to $160 / 1000$ population in 2018 but radically decreased to $140 / 1000$ population in 2019 due to some interventional activities 
Plasmodium specious varies from woreda to woreda. distribution of Plasmodium ( ratio of plasmodium Falciparum to Plasmodium vivax) in Ankesha Guagusa, Banja, Guagusa Shekudad, Changi, and Zigem were $75: 25,51: 49,88: 12,72: 28$ and $86: 14$ respectively, and the total zonal ratio is 76:24 which is relatively matching with the national standard of the Plasmodium distribution of falciparum versus vivax, which is 65: 34 (Anam et al., 2019).

However, in some woredas, the distribution of Plasmodium vivax species is higher than that of the spreading of falciparum which not matched the national guideline standards. These woredas include Guagusa Shekudad and Zigem. It is agreed with the study conducted at the Butajira area, which dedicated an increased proportion of Vivax malaria at high altitudes, the decrease in Vivax malaria in the highland-fringe area and the high transmissibility of P.vivax. The P. falciparum prevalence increased by 75\% in 2015 to $86 \%$ in 201/2015, and higher than the national report 2019 (Delil et al., 2016).

This study provided that the following woredas, for example, Ankesha Guagusa, Banja, Guagusa Shikudad, Changi, and Zigem had the mean yearly anticipated occurrence of definite malaria higher than twenty per thousand human, this may be due to the higher number of reporting sites, arid areas and the presence of agricultural programs could affecting diseases spread where incidence be higher than expected and the rest of woreda be constantly under 5 reported malaria per thousand population per year (Rabbani et al., 2016). In general, the utilize of prevalence while contrasting between woreda get better the capability of the zone to map property properly, develop targeted diseases organize labor, and permit an improved assessment of the program.

There have been no well-organized epidemic preparedness and response planning, and no financial and/ or support. This could cause weak case detection and response during epidemics. Preparedness aims to strengthen capacity in recognizing and responding to public health emergencies through conducting regular risk identification and analysis, establishing partnerships and relationships, improving community participation, and implementing community-based interventions and strategic communication during the pre-emergency phase and ensuring their monitoring and evaluation (FDRE Public Health institute, 2012).

\section{Limitation Of The Study:}

Small sample size and Regional one due to resources shortage.

\section{Conclusions}

The current PHS study revealed that the malaria incidence rate showed a remarkable decline. However, the annual parasitic incidence rate remains constant. This indicates that ivermectin did not affect malaria elimination.

The finding suggests the importance of PHS training for focal persons and strict follow-up of the implementation of the acceptable surveillance system might improve PHEM capacity. Besides, minimizing the irrational case definition of diseases could also help to improve PHEM capacity. 


\section{Abbreviations}

EFY: Ethiopian Fiscal Year, PHS: Public Health Surveillance, PH: Public Health, HO: Health Office, HC: Health Center, PV: Predictive Value, HP: Health Post, PHEM: Public Health Emergency Management.

\section{Declarations}

Ethics approval and consent to participate: Ethical clearance was obtained from the Institutional Review Board of the Jimma University and an official letter was submitted to the concerned bodies. The concerned bodies were informed to get the assurance of the study and confidentiality was maintained at all levels of the study. Informed consent was obtained from all participants and the Institutional Review Board of the Jimma University approved it with Ethical approval of Research protocol letter with its reference number IRB00010/2020.

\section{Informed consent}

Dear Sir/madam;

My name is and I am from I am conducting data collection on the Trends of Malaria on Hotspot and Ivermectin Mass-drug Administration Zone of Amhara Regional State, 2020. The study being conducted by Mr. Chalachew Yenew from Jimma University.

You are kindly requested to be included in the study, which has great importance in improving health. The interview will take a maximum of minutes.

No information concerning you as an individual will be passed to another individual or institution. Your participation will be based on your willingness and you have the right not to participate fully or partially. If you agree to be included in the study, I will start my question by asking general identification questions.

1. Agree to participate Do not Agree to participate

Thank you for your cooperation!!!

Name of data collector Date Signature

Name of the supervisor Date Signature

Consent to Publish: Not applicable

Availability of data and materials: All data generated and analyzed during this study are included in this manuscript itself. 


\section{Competing interests: The authors have announced that they have no competing interests in nonfinancial includes political, personal, religious, ideological, academic, and intellectual and Financial issues.}

\section{Funding: Not Applicable}

\section{Authors' contribution}

SM and AA are actively involved during the conception of research issues and the development of research proposals and $\mathrm{CY}$ has been writing various parts of the research report and prepares the final manuscript. All authors read and approved the final manuscript.

Acknowledgments

Firstly, we would like to give our heartfelt thanks to the almighty God for giving us the knowledge and the support we need to do this study.

We also would like to acknowledge the Jimma and Debre Tabor University, College of Health Sciences for the arrangement and administrative support of our study.

\section{References}

1. Anam LS, Badi MM, Assada MA, Al Serouri AA. (2019). Evaluation of Two Malaria Surveillance Systems in Yemen Using Updated CDC Guidelines: Lessons Learned and Future Perspectives. Inquiry (United States), 56. https://doi.org/10.1177/0046958019880736.

2. Chaccour C, Hammann F, Rabinovich NR. (2017). Ivermectin to reduce malaria transmission I. Pharmacokinetic and pharmacodynamic considerations regarding efficacy and safety. Malaria Journal, 1-16. https://doi.org/10.1186/s12936-017-1801-4.

3. Chala B. (2020). Five Year Trend Analysis of Malaria Cases in Adama, Boset and Lume Districts of East Shawa ,.

4. Delil RK, Dileba TK, Habtu YA, Gone TF, Leta TJ. Magnitude of malaria and factors among febrile cases in low transmission areas of Hadiya Zone, Ethiopia: A facility based cross sectional study. PLoS ONE. 2016;11(5):1-17. https://doi.org/10.1371/journal.pone.0154277.

5. FDRE Public Health institute. (2012). Public Health Emergency Management. 1-140.

6. German RR, Lee LM, Horan JM, Milstein RL, Pertowski CA, Waller MN. \& Guidelines Working Group Centers for Disease Control and Prevention (CDC). (2001). Updated guidelines for evaluating public health surveillance systems: recommendations from the Guidelines Working Group. MMWR. Recommendations and Reports: Morbidity and Mortality Weekly Report. Recommendations and Reports, 50(RR-13), 1-35; quiz CE1-7. http://www.ncbi.nlm.nih.gov/pubmed/18634202. 
7. Rabbani F, Shipton L, Aftab W, Sangrasi K, Perveen S, Zahidie A. Inspiring health worker motivation with supportive supervision: A survey of lady health supervisor motivating factors in rural Pakistan. BMC Health Services Research. 2016;16(1):1-8. https://doi.org/10.1186/s12913-016-1641-x.

8. The Carter Center. (2012). 3rd ANNUAL MALARIA CONTROL PROGRAM REVIEW Ethiopia and Nigeria Held on February 24, 2012 at The Carter Center, Atlanta, Georgia. 2012, September 2012.

9. World Health Organization. An operational manual. Malaria Treatment Gudelines. 2013;2:3-68. (www.who.int/malaria).

10. World Health Organization. (2018). Malaria Surveillance, Monitoring \& Evaluation: a Reference Manual.

11. World malaria report. (2019). World malaria report 2019. In WHO Regional Office for Africa. https://www.who.int/news-room/fact-sheets/detail/malaria.

\section{Figures}

\begin{tabular}{|c|c|c|c|c|c|c|}
\hline \multicolumn{7}{|l|}{$120 \%$} \\
\hline \multicolumn{7}{|l|}{$100 \%$} \\
\hline \multicolumn{7}{|l|}{$80 \%$} \\
\hline \multicolumn{7}{|l|}{$60 \%$} \\
\hline & & & & & & \\
\hline \multirow{3}{*}{$\begin{array}{r}20 \% \\
0 \%\end{array}$} & & & & & & \multirow{2}{*}{$\begin{array}{l}\text { Completeness } \\
\text { EWHO standard }\end{array}$} \\
\hline & & & & & & \\
\hline & $\begin{array}{l}\text { Ankasha } \\
\text { Guagusa }\end{array}$ & Banja & Chagni & $\begin{array}{l}\text { Guagusa } \\
\text { Shekudad }\end{array}$ & Zigem & \\
\hline Completeness & $96 \%$ & $97 \%$ & $100 \%$ & $95 \%$ & $95 \%$ & \\
\hline WHO standard & $80 \%$ & $80 \%$ & $80 \%$ & $80 \%$ & $80 \%$ & \\
\hline
\end{tabular}

\section{Figure 1}

The open-source statistical program $\mathrm{R}$ graphical outputs of the timelines \& completeness of the reports of different woreda of Awi Zone, Amhara Regional State, Ethiopia, 2020. 


\section{Malaria Average Annual Parasitic incidence}

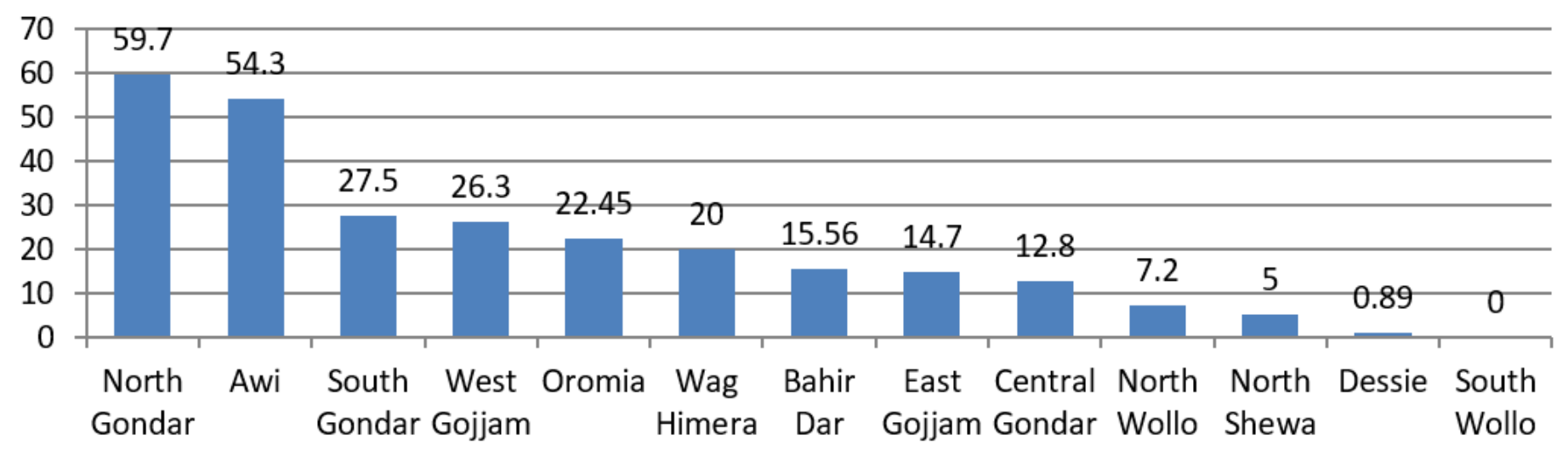

Figure 2

The open-source statistical program R Graphical output of malaria average annual parasitic incidence rate per 1000 population by zones in Amhara Region, Ethiopia, 2015-2019.

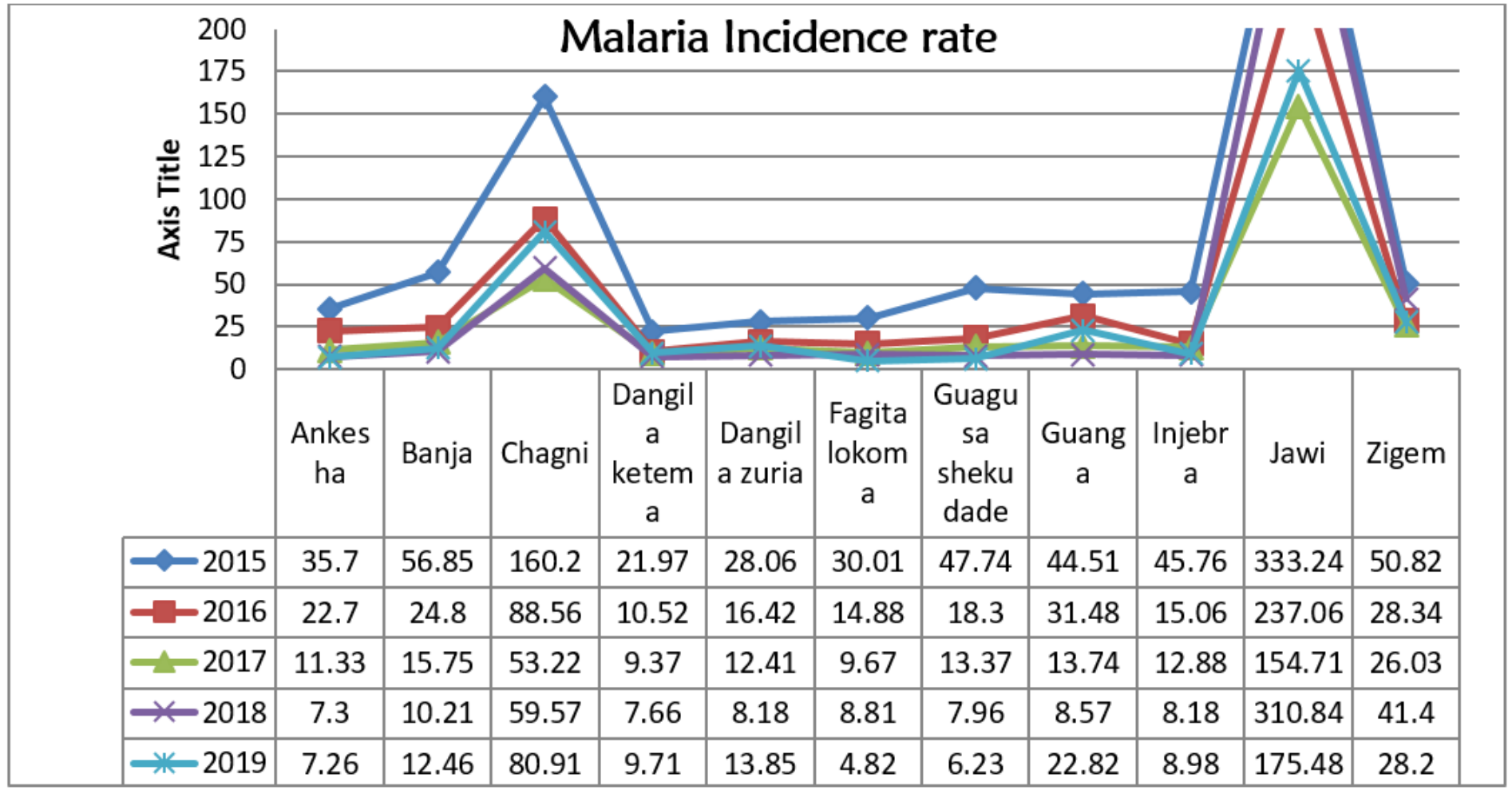

Figure 3

The open-source statistical program $\mathrm{R}$ graphical outputs of the spatiotemporal distribution of malaria incidence by Years, Woreda of Awi Zone, Amhara Region, 2020. 


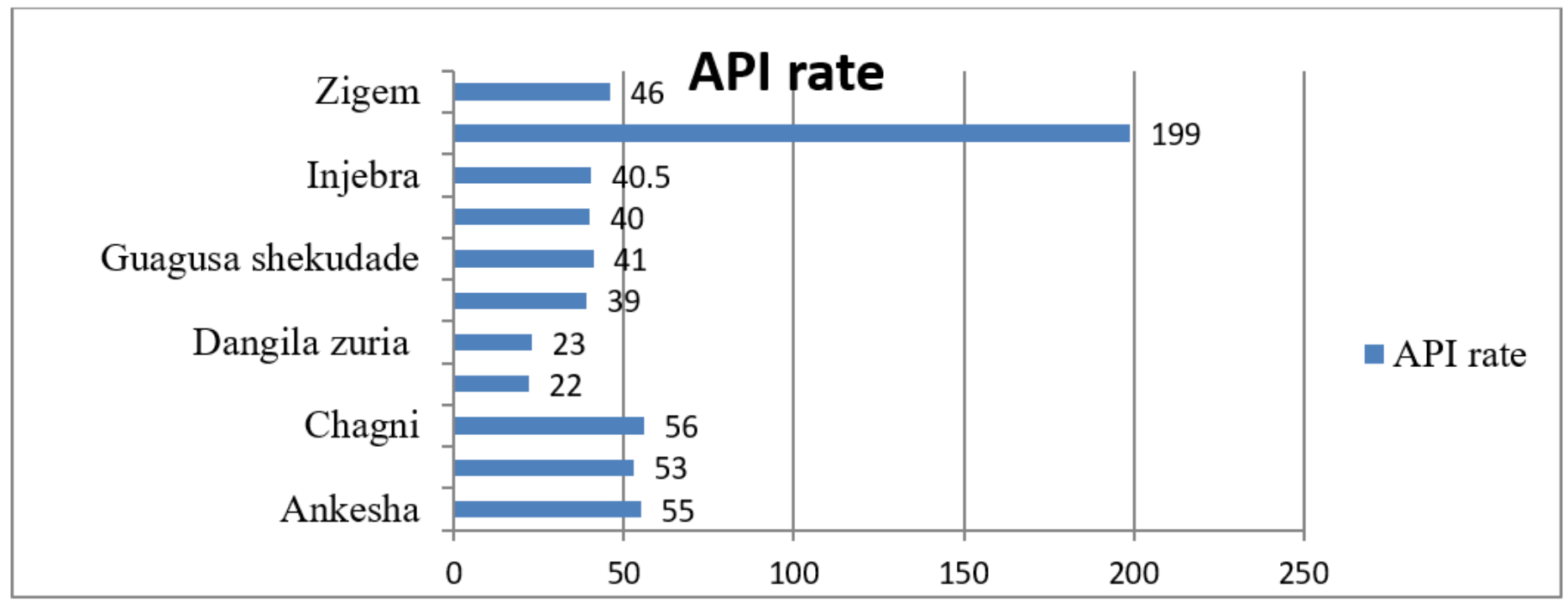

Figure 4

The open-source statistical program R graphical output of API rate of the study area, Awi Zone, Amhara Region, 2019.

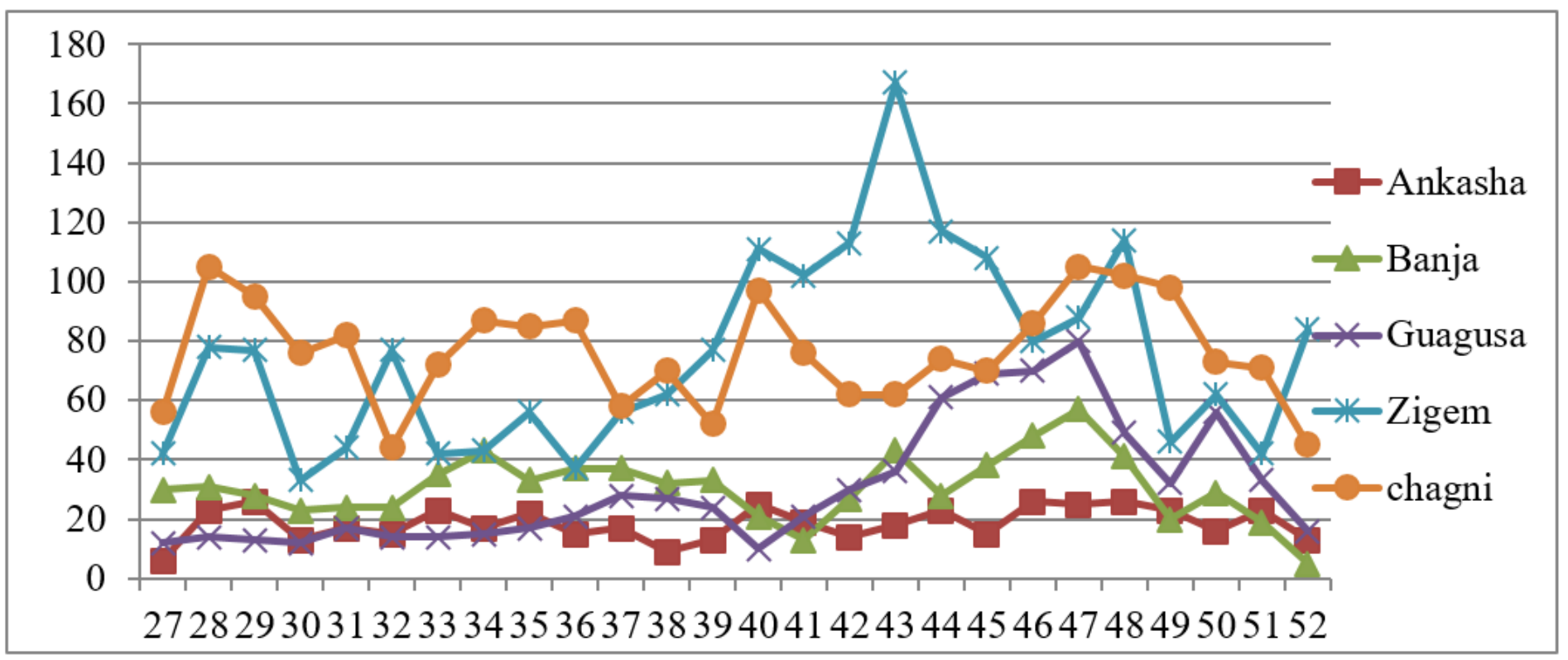

Figure 5

The open-source statistical program R graphical outputs of trends of confirmed malaria cases by woreda and WHO Epidemiologic week of Awi Zone, woreda, Amhara region Ethiopia, 2020. 